Über Kurven und Flächen in Allgemeinen Räumen. By P. Finsler. A reprint of the 1918 dissertation with a detailed bibliography by H. Schubert. Basel, Birkhäuser, 1951. 12+160 pp. 14.80 Swiss fr.

This unaltered photographic reproduction of Finsler's thesis will be welcome to many mathematicians. For, as Ostrowski states in his preface, this is one of the few first works which have had a great and lasting influence, and, being a thesis, existed in only very few copies many of which are owned by Finsler's friends. But the book will be more than a classic in a geometer's library. The reviewer concludes from his own sad experience that there must be many instances where apparently new results were found to be already in Finsler's thesis after the latter finally became available through interlibrary loan. Moreover, there are many ideas which may facilitate later work. An example, where this could have, but did not, happen because the thesis was so inaccessible, is furnished by the expression (58), p. 59, for curvature, which can be applied without change to general metric spaces, and was later proved by Haantjes to be essentially equivalent to Menger's curvature. In addition to the thesis the present edition contains a comprehensive list of books and papers concerning Finsler spaces (until 1949) compiled by H. Schubert. It has a wide scope and comprises references to tensor calculus, ordinary and Riemannian geometry, the geometry of paths, the calculus of variations, spaces of infinite dimension, which are ordinarily not considered as contributions to Finsler spaces. This bibliography will prove very helpful, provided the user is aware of the fact that it is not complete, even as to papers which contain results on Finsler spaces in the narrow sense.

\title{
H. BusemanN
}

Integral transforms in mathematical physics. By C. J. Tranter. New York, Wiley, 1951. $10+118$ pp. $\$ 1.50$.

A variety of linear integral transformations that are useful in solving boundary value problems in differential equations is presented briefly here. In addition to the usual Laplace, Fourier, Mellin, and Hankel transformations, finite Fourier, Hankel, and Legendre transformations are considered. The inverses of those transforms are given, accompanied in most cases by formal derivations. Applications of those transformations to the solution of boundary value problems in partial differential equations of physics or engineering are illustrated. Short chapters are included on numerical evaluation of integrals and on a combination of relaxation and transformation 
methods. Each transformation reduces a characteristic differential form in the original function to an algebraic form in the transform. The algebraic form also involves characteristic boundary values of the original function. Those characteristic forms and boundary values determine the types of boundary value problems that can be solved directly by the particular transformation. They are indicated here, sometimes not explicitly, but they are not emphasized. In fact, at the beginning of the second chapter the author states that his examples will serve as a guide in selecting appropriate transforms for the solution of boundary value problems. Some of the examples presented display no advantage in using the transformation that is being illustrated, or in using any transformation. The book gives some good indications of the importance of various integral transformations in the solution of boundary value problems; it could have presented a still more convincing case for such transformations.

\section{R. V. Churchill}

Atti del Terzo Congresso dell' Unione Matematica Italiana tenuto in Pisa nei giorni 23-26 Settembre 1948. Rome, Edizioni Cremonese della Casa Editrice Perrella, 1951. 4+265 pp. 1600 lire.

This volume contains lectures by Severi, Terracini, Martinelli, Cesari, Agostini, and Tolotti, and about 100 abstracts of papers presented to the Congress.

Opere. By Felice Casorati. Vol. 1. Rome, Edizioni Cremonese della Casa Editrice Perrella, 1951. 16+420 pp. 4000 lire.

This volume, prepared by the Unione Matematica Italiana, contains a bibliography, a life by E. Bertini, two lectures, three papers on geodesy, and fourteen papers on the theory of functions of a complex variable.

Moderna teoria delle funzioni di variabile reale. By G. Vitali and G. Sansone, 3d ed. Part 1, by Giuseppe Vitali. Aggregati, analisi delle funzioni, integrazione, derivazione. Bologna, Zanichelli, 1951. 8+222 pp. 3000 lire.

The first edition (1935) was reviewed in Bull. Amer. Math. Soc. vol. 42 (1936) p. 15. Little change has been made in the later editions. 